\title{
Advanced decision and intelligence technologies for manufacturing and logistics
}

\author{
Chen-Fu Chien · Kap Hwan Kim • Baoding Liu • \\ Mitsuo Gen
}

Published online: 27 July 2011

(C) Springer Science+Business Media, LLC 2011

Manufacturing and logistics are globally facing transformational changes. The introduction of new technologies and the advances in equipment and intelligence capability are having profound effects on production and logistics in practice. New solutions for manufacturing and logistics are evolving to respond to various needs. In particular, production and services are transformed from vertically integrated firms for product-based business into virtual collaborations of horizontal modular partners, while huge amounts of data are increasingly accumulated due to the business integration. Recently, soft computing and decision technologies have developed to analyze field data and to generate intelligent algorithms that enable automated manufacturing and logistics systems to control the work flow, material flow, and information flow of global supply chain networks based on IC tag. By the seamless integration of intelligence and decision technologies combined with IT technology, Intelligent Manufacturing \& Logistics Systems have completely changed the way we manage our factories, logistics, outsourcing, and supply

\section{C.-F. Chien $(\varangle)$}

Department of Industrial Engineering and Engineering

Management, National Tsing Hua University, Hsinchu 30013,

Taiwan

e-mail: cfchien@mx.nthu.edu.tw

\section{K. H. Kim}

Department of Industrial Engineering, Pusan National University, Jangjeon-dong, Geumjeong-gu, Busan 609-735, South Korea

e-mail: kapkim@pusan.ac.kr

\section{B. Liu}

Department of Mathematical Sciences, Tsinghua University, Beijing 100084, China

e-mail: liu@tsinghua.edu.cn

M. Gen

Fuzzy Logic System Institute, Iizuka-shi, Fukuoka, Japan

e-mail: gen@flsi.or.jp chain networks. This Special Issue of the Journal of Intelligent Manufacturing (JIM) aims to address the critical issues involved Advanced Decision and Intelligence Technologies for Manufacturing and Logistics

The purpose of this special issue is to promote the exchange of information and disseminate the research results on advanced decision and intelligence technologies for manufacturing and logistics. The intent of the special issue is to enable the researchers and practitioners in this field to keep up with these fast advanced intelligence techniques and to promote a better understanding and collaboration among them. The special issue presents insightful, comprehensive, theoretical, practical and real-world applications of advanced decision and intelligence technologies in manufacturing and logistics. Original, high quality papers and empirical research papers with scientific novelty were invited from scholars and practitioners to address this subject. This special issue was addressed to participants of International Conference on Intelligent Manufacturing \& Logistics Systems (IML2010), February 27-March 01, 2010 at the Hsinchu, Taiwan. All manuscripts are recommended by session chairs of IML2010 conference, while other papers are also directly submitted for review and publication. All the submitted manuscripts were carefully blind peer reviewed by at least two referees, in accordance with Journal of Intelligent Manufacturing procedure and finally fifteen papers were selected from many submissions for this special issue.

The papers in this issue can be clustered into four interrelated groups to address the theme of this special issue from various perspectives. The first group addresses the important issues for advanced decision methodologies for manufacturing. In particular, "A hybrid fuzzy AHP-PROMETHEE decision support system for machine tool selection in flexible manufacturing cell" by Taha and Rostam, who present a hybrid approach of fuzzy analytic hierarchy process 
(fuzzy AHP) and preference ranking organization method for enrichment evaluation (PROMETHEE) to select the best alternative machine. This study has been applied to computer numerical controlled (CNC) turning centre machine for a flexible manufacturing cell (FMC).

"Mini-max regret strategy for robust capacity expansion decisions in semiconductor manufacturing" by Chien and Zheng is a mini-max regret strategy for capacity planning under demand uncertainty to improve capacity utilization and capital effectiveness for healthy growth in semiconductor manufacturing. The proposed approach considers possible outcomes of the multi-period demand forecasts from sales and marketing teams to minimize the maximum regrets of capacity oversupply and shortage and thus derive a robust capacity expansion plan along with the time. An empirical study conducted in a leading semiconductor company in Taiwan has validated this approach.

"An efficient algorithm for stochastic capacity portfolio planning problems" by $\mathrm{Wu}$ and Chuang, investigates the capacity portfolio planning problems under demand, price, and yield uncertainties and models such problems as Markov decision process (MDP) by considering two types of capacity: dedicated and flexible capacity. In order to solve the large stochastic optimization problems, a new heuristic search algorithm (HSA) is developed to reduce computational complexity. Based on the numerical problems generated by experimental design techniques, HSA reduces computational time by at least $30 \%$ in large capacity optimization problems.

"Decision-making for the best selection of suppliers by using minor ANP" by Ozaki, Lo, Kinoshita, and Tzeng, describes using Minor ANP to facilitate priority selection of suppliers by irregular ANP with missing values or the non-square matrix. The authors also showed that the missing values in the alternative matrix can be replaced by zeros and the criteria matrix can be derived without using the inverse alternative matrix. The second group focuses on developing meta-heuristic algorithms and applications in logistics and transportation planning.

The second group solves critical problems involved in intelligent logistics with both academic and industrial importance. "Queue-based local scheduling and global coordination for real-time operation control in a container terminal" by Choe, Cho, Park, and Ryu, proposes a queue-based local scheduling and global coordination method to optimize and synchronize the operations of various equipment for maximizing productivity of a container terminal. The results of simulation experiment show significant improvement on the synchronization between yard tractors and stacking cranes under a quay cranes delay.

"Workload-based yard-planning system in container terminals" by Won, Zhang, and Kim, develops an integrated decision-making framework for the yard-planning and simul- taneously considers various resources such as storage space, yard cranes, and traffic area in the container terminals. The yard-planning is extended to integrate with crane development.

"A berth allocation planning problem with direct transshipment consideration" by Liang, Hwang, and Gen, introduces the Berth Allocation Planning (BAP) problem by considering the transshipment of ship to ship and formulates a mathematical model with different number of Quay Cranes (QCs) in the berth. A hybrid multistage operationbased Genetic Algorithm (h-moGA) with a priority-based encoding method is proposed for solving BAP problem. The computation experiment reveals that direct transshipment is better when the time gap between two ships is rather small and the quantity of transshipment is large, and then the service time can be reduced.

"Retailing policy for perishable item sold from two bins with mixed issuing policy" by Han, Oh, and Hwang, develops a mathematical model involving Last-In-First-Out (LIFO) and First-In First-Out (FIFO) issuing policy to maximize the retailer's profit, and a solution procedure based on Tabu search is applied to solve problems. A numerical example is used for validation, and a sensitivity study is performed in the practical cases.

"Design of an improved quantum-inspired evolutionary algorithm for a transportation problem in logistics systems" by Wang, Kowk, and Ip, extends the standard of QuantumInspired Evolutionary Algorithm (QEA) by combining its principles with some heuristics methods, which is applied to optimize the vehicle routing problem with time windows (VRPTW). Their computational result of the simulation problem shows that IQEA can achieve a relatively optimal solution.

The third group focuses on complex challenges in modern production facilities for manufacturing scheduling and production planning. In particular, scheduling is one of the most important fields in advanced planning scheduling (APS) for manufacturing optimization. "Network modeling and evolutionary optimization for scheduling in manufacturing" by Lin, Hao, Gen, and Jo, proposes a multi-section evolutionary algorithm for the scheduling models formulated by network modeling. In their study, the auto-scheduling in the manufacturing system and service-oriented evolutionary computation architecture (SoECA) software is introduced to improve evolutionary computation's availability.

"A novel bi-vector encoding genetic algorithm for the simultaneous multiple resources scheduling problem" by Wu, Hao, Chien, and Gen, develops a genetic algorithm (bvGA) incorporating a novel bi-vector encoding method representing the chromosomes of operation sequence and seizing rules for resource assignment in tandem. Their experiment study reveals that computation time and solution quality can be improved by using bvGA. 
"Scheduling two-stage hybrid flow shops with parallel batch, release time, and machine eligibility constraints" by Wang, Yang and Chang, proposes an efficient dispatching rule called BFIFO that assigns jobs or batches to machines based on first-in-first-out (FIFO) principle, and then provides several re-optimization techniques using MIP and local search heuristics involving interchange, translocation and transposition (ITT) among assigned jobs. The result shows the effectiveness of using BFIFO+ITT to solve difficult scheduling problems in real-world semiconductor manufacturing.

The fourth group proposes novel approaches for intelligence technologies and applications in various domains. In particular, "Manufacturing intelligence to forecast and reduce semiconductor cycle time" by Chien, Hsu and Hsiao, develops a manufacturing intelligence approach by integrating Gauss-Newton regression (GNR) method and adopts back-propagation neural network (BPNN) as basic model to forecast the production cycle time in a semiconductor fab, in which WIP, capacity, utilization, average layers, and throughput are rendered as input factors for indentifying effective rules. The authors also develop an adaptive model for rapid response to the change of production line status to control the levels of the corresponding factors to reduce the cycle time. Based on the empirical study from a wafer foundry, the results demonstrate the effectiveness by reducing the forecast errors in the dynamic operation conditions to thus reduce the cycle time effectively.
"Bullwhip Effect Measure in a Seasonal Supply Chain" by Cho and Lee, derives lead-time demand forecast, forecast error, and the optimal inventory policy for the retailer to measure the bullwhip effect in a seasonal supply chain. The result finds that the replenishment lead time must be less than the seasonal cycle in order to reduce the bullwhip effect.

"Intelligence modeling for coping strategies to reduce emergency department overcrowding in hospitals" by Chan, Huang, and You, proposes an intelligent model which first introduces the well-known decision tree method to fit an accommodated nonlinear association and to obtain intelligent grading rules of emergency department (ED) crowding; Then it integrates the intelligent grading rules and indexes of coping strategies to construct a hierarchical linear model (HLM). The proposed approach is validated by academic medical centers of Taiwan and is useful in providing information to National Health Insurance (NHI) administrators by putting relevant strategies or policies into action to solve ED crowding.

The publication of this special issue is dependent on the cooperation of the authors, reviewers, and editors. We would like to take this opportunity to express our sincere appreciation to all of the authors, reviewers, the Editor-in-Chief Dr. Andrew Kusiak of Journal of Intelligent Manufacturing, and the Executive Guest Editor, Dr. Chia-Yu Hsu, for their invaluable contributions to this special issue. 